\title{
Separation and Analysis of Soluble Organic Compounds from Corncob Guizhen Gong ${ }^{a}$, Rui Sun ${ }^{b}$ and Wen $X u^{c}$ \\ School of Chemical Engineering, Xuzhou University of technology, Xuzhou 221018, China \\ a ggz72@163.com, b yaven.sytark@qq.com, c 2869280023@qq.com
}

Keywords: Corncob; Acetone; Petroleum Ether; FTIR

\begin{abstract}
The stepwise extractions of corn cob were carried out with petroleum ether and acetone, respectively. The extracts were detected by FTIR. The results show that the dissolution law of organic matter in two solvents is similar. In the fourth extract, the content of the substance was relatively weak and increased again fifth times. The main functional groups of the two solvent extracts are ether, alcohol, carbonyl group, chlorine substitute and aromatic compounds. The polar substance containing oxygen is more easily extracted by acetone. The presence of an absorption peak at $615 \mathrm{~cm}^{-1}$, indicating the presence of chlorine in the corncob. Some components are not extracted in the residue.
\end{abstract}

\section{Introduction}

In China, Corn is the first agricultural products, the total planting area is about 1249.35 square kilometers, there are about 1.5 tons annual output and about 40 million tons of corncob are produced each year. Some of these corn cobs were collected as feed or culture medium, and large amounts of which were discarded or burned directly. Not only waste of resources, but also cause certain air pollution. Corncob consists of cellulose, hemicellulose and lignin[1]. Some uses have been developed by some countries[2-6]. The key problem affecting its processing and utilization is the insufficient understanding of its organic structure.

In order to fully understand its molecular structure from molecular level, corn cob was extracted with petroleum ether and acetone. The extracts were analyzed with FTIR. This study provides a theoretical basis for its high value-added use.

\section{Experimental}

Materials. Corncob used in this study was collected from Xuzhou city, Jiangsu, China. It was washed with de-ionized water, and air-drying, then, which was ground to pass through 200 mesh sieve. All the raw materials were dried in an oven at $100 \mathrm{oC}$ for $5 \mathrm{~h}$, and then stored in a desiccator at room temperature before using. Acetone and petroleum ether used in the experiment are analytical grade, obtained from the commercial source, and were used without further purification.

Experimental Method. $2 \mathrm{~g}$ Corncob and $100 \mathrm{~mL}$ acetone were put into Soxhlet extractor. The Soxhlet extractor was heated up to boiling point and kept at the temperature for $5 \mathrm{~h}$, then cooled down to room temperature. The extracts mixture was taken out from the

extractor. The extracts were distilled using a Büchi R-134 rotary evaporator to afford acetone extract (AE), which was analyzed with FTIR. The extracted residue was dried in a vacuum drying box and weighed. Then the next extraction were carried out, successively followed for 5 times. Petroleum ether extraction took the same process as acetone.

The extracts gained were analyzed by FTIR spectroscopy (Alpha, Bruker company, German) using potassium bromide tableting method over a scan range of 500 to $4000 \mathrm{~cm}^{-1}$.

\section{Results and Discussion}

FTIR Analysis of Extracts. FTIR of acetone extract (AE) and petroleum ether extract is shown in Figure 2. As shown in Figures, $3417 \mathrm{~cm}^{-1}$ is $-\mathrm{OH}$ absorption peak, which is stronger in the two solvent extracts, and the association degree decreases in turn, showing that hydroxyl content was 
higher in the two solvent extracts. The absorption peak at $2969 \mathrm{~cm}^{-1}$ is assigned to aliphatic C-H, and the intensity decreases as the extraction number increase. The absorption peak of $1733 \mathrm{~cm}-1$ is attributed to $\mathrm{C}=\mathrm{O}$ group, and the absorption of two solvents is weaker in the fourth times. The absorption peak at $1637 \mathrm{~cm}^{-1} 1618 \mathrm{~cm}^{-1}$ is benzene ring skeleton $\mathrm{C}=\mathrm{C}$ stretching vibration. At 1162 $\mathrm{cm}^{-1}$, the absorption peak is $\mathrm{C}-\mathrm{O}$, which increased with the increase of extraction times. The absorption peak at $615 \mathrm{~cm}^{-1}$ is the $\mathrm{C}-\mathrm{Cl}$ absorption peak, and the presence of this peak indicates evidence of chlorine in the corncob.

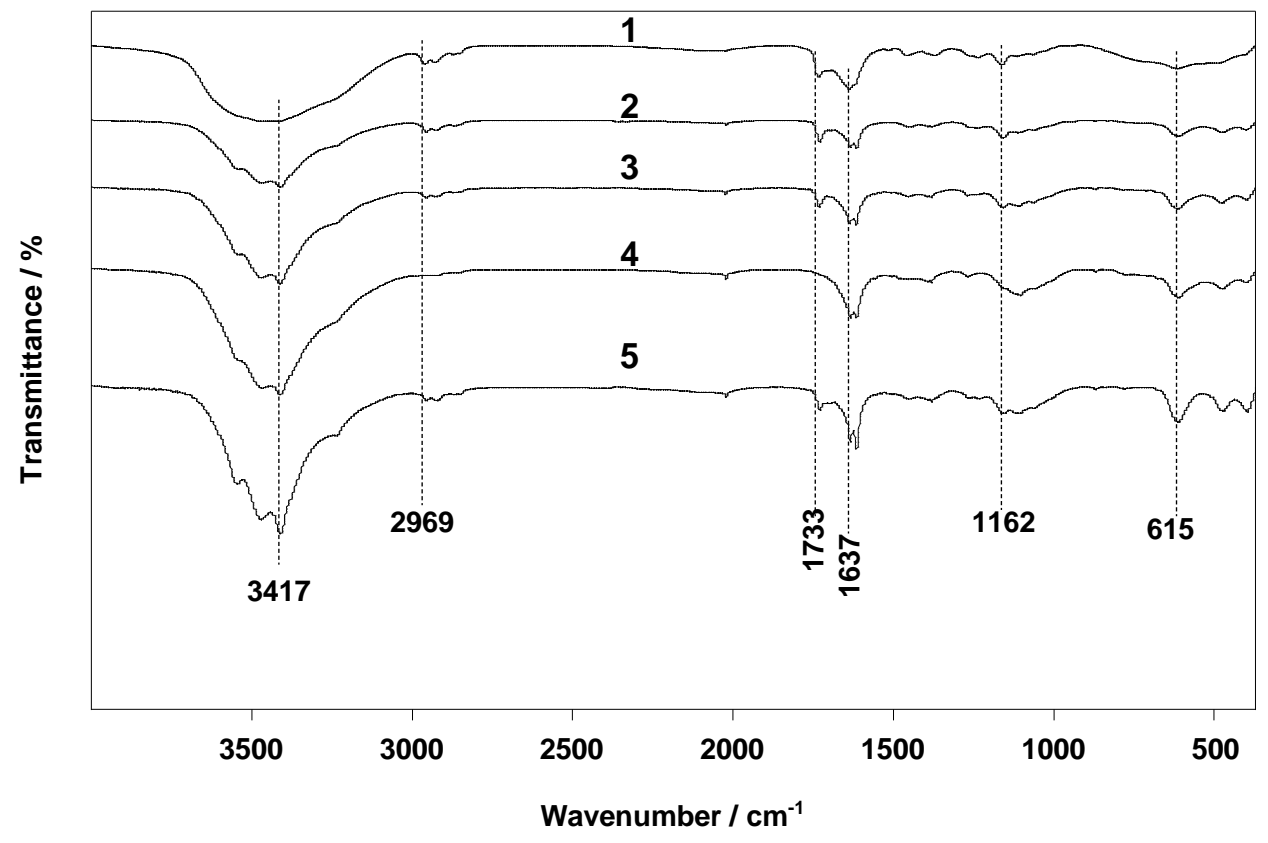

Fig. 1. FTIR spectra of acetone extraction

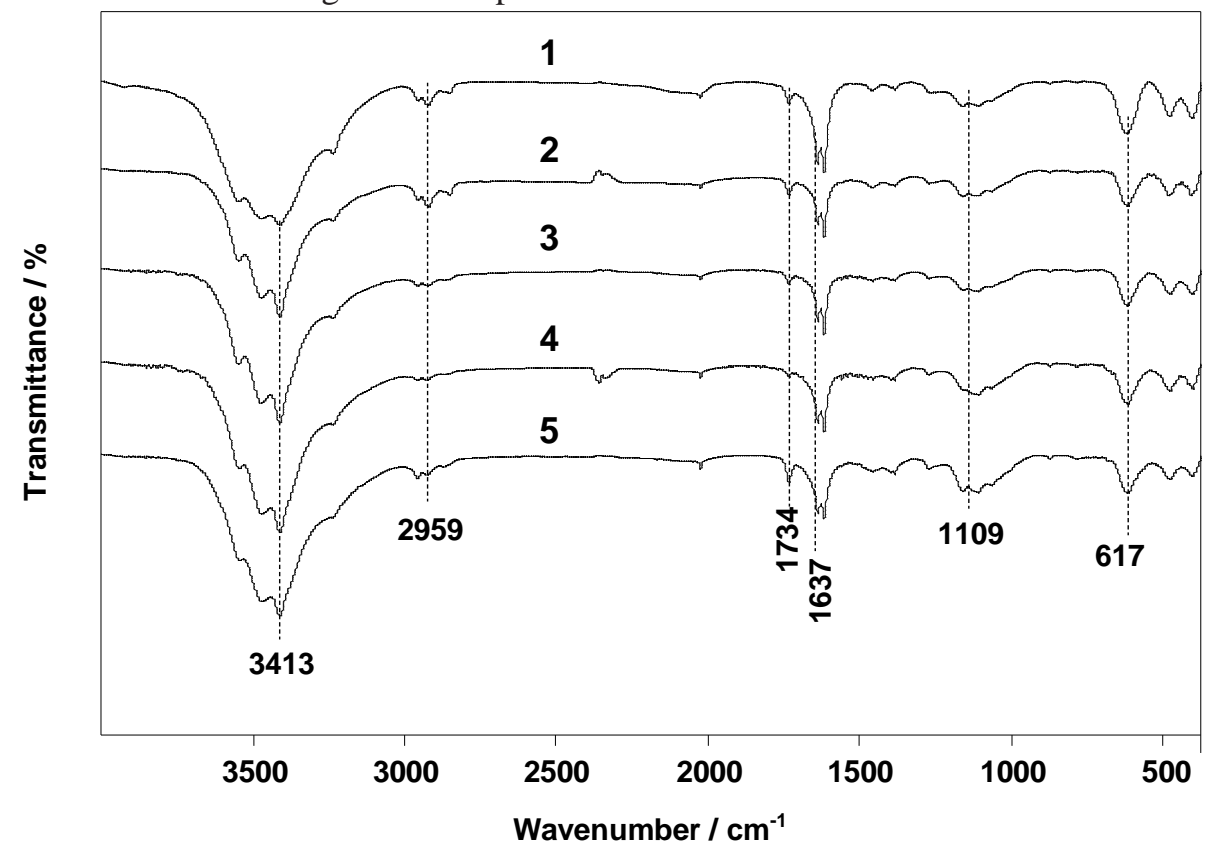

Fig. 2. FTIR spectra of PE extraction

Fig. 3 is FTIR spectra of acetone residue (AR) and petroleum ether residue (PR). As shown in Figure, the major characteristic peaks are similar to those of the extracts, showing that some components are not extracted in the residue. $1734 \mathrm{~cm}^{-1}$ absorption and C-O absorption peak at 1050 $\mathrm{cm}^{-1}$ acetone residue are weaker than those of petroleum ether, because acetone polarity is greater than petroleum ether, according to the principle of similar dissolution, oxygen polar material is easier to be extracted by acetone. 




Fig. 3. FTIR spectra of AR and PR

\section{Summary}

The stepwise extractions of corn cob were performed using petroleum ether and acetone respectively. The dissolution law of organic matter in two solvents is similar. In the fourth extract, the content of the substance was relatively weak and increased again fifth times, indicating that after four extraction to fifth times, there may be some changes different from the first 4 times, so that more substances dissolved. The polar substance containing oxygen is more easily extracted by acetone. The main functional groups of the two solvent extracts are ether, alcohol, carbonyl group, chlorine Substitute and aromatic compounds. The presence of an absorption peak at $615 \mathrm{~cm}^{-1}$, indicating the presence of chlorine in the corncob.

\section{Acknowledgement}

This work was supported by the China Building Material Federation (2014-M3-4), Xuzhou Information Institute (XKQ016), Innovative Training Program for College Students of Xuzhou University of Technology (XCX2017155), Innovation and Entrepreneurship for College Students of Xuzhou University of Technology (2017042) and Open laboratory project of Xuzhou University of Technology.

\section{References}

[1] C.G. Boeriu , D. Bravo, R.J.A. Gosselink and J.E.G. vanDam, Characterization of structure-dependent functional properties of lignin with infrared spectroscopy, Industrial Crops and Products, 4 (2004) 205-218.

[2] L.F. Gao, H.B. Xu, Y. Zhang and H. B. Cao, Optimization on production process of furfural by high-temperature dilute-acid hydrolysis of corncobs, The Chinese Journal of Process Engineering, 10 (2010) 292-297. (In Chinese)

[3] P. Benar, A.R. Goncalves, D. Mandelli and U. Schuchardt, Eucalyptus or ganosolv lignins: study of the hydroxymethylation and use in resols, Bioresource Technology, 68 (1999) 11-16.

[4] J.A. Velasquez, F. Ferrando and J. Salvado. Effects of Kraft lignin addition in the production of binderlessfiberboard from steam exploded Miscanthus sinensis, Industrial Crops and Products, 18 (2003) $17-23$.

[5] A. M. Boudet. S. Kajita, J.G Pettenati and D. Goffner, Lignins and lignocellulosics: a better control of synthesis for new and improved uses, Trends in Plant Science, 12 (2003) 576-581. 
[6] R.K. Sharma, J.B. Wooten, V.L. Baliga, X.H. Lin, W. Geoffrey Chan and M.R. Hajaligol, Characterization of chars from pyrolysis of lignin, Fuel, 83 (2004) 1469-1482. 